\title{
sciforum
}

Conference Proceedings Paper

\section{First Conclusions from the WMO/GAW Coordinated Study on Impacts of COVID-19 Lockdown Measures on Air Quality: An Observational and Modelling Analysis}

\author{
Ranjeet S Sokhi, Vikas Singh, Xavier Querol, Sandro Finardi, Maria Fatima Andrade, Rebecca \\ Garland, Radenko Pavlovic, Admir Admir Créso Targino, Shaofei Kong, Kester Momoh, \\ Rajasree Meethal, Sabine Fritz, Push Raj Tiwari, Marc Guevara, Hugo Denier van der Gon, \\ Vincent-Henri Peuch, Greg Carmichael, Oksana Tarasova, Alexander Baklanov, Lu Ren and \\ many global contributors
}

* Correspondence: r.s.sokhi@herts.ac.uk

\begin{abstract}
The aim of this World Meteorological Organisations' Global Atmospheric Watch (WMO/GAW) programme coordinated study is to understand how air quality has changed in cities across the globe as a result of measures to control the spread of the COVID-19 virus. The observational analysis reported here focusses on changes in PM2.5, PM10, NO2 and O3 concentrations during the 2020 lockdown periods in more than forty cities compared to the mean of previous five year (2015-2019) for the same periods. Similarly, a range of meteorological parameters have also been compared to assess how the meteorology may be different between the 2020 and the previous five years. All observational data has been supplied by local teams who were responsible for the QA/QC checks. A new phase of modelling analysis has also been initiated and we report here on one case study for the changes in air pollution over the UK. For the modelling analysis, a baseline (BL) was defined to represents the UK and Europe emissions assuming no lockdown measures between 1 March to 26 April 2020 (with lockdown beginning on 24 March). Scenario 1 (S1) is defined as a plausible scenario to represents the overall comprehensive changes in emissions in the key sectors over the lockdown period of 24 March to 26 April. A second scenario (S2) represents a sensitivity scenario to estimate the changes in air quality species attributable to reductions only in road traffic emissions over the lockdown period of 24 March to 26 April. The modelling analysis has been conducted with WRF/CMAQ modelling system with a spatial grid resolution of $10 \times 10 \mathrm{~km}$ over the UK domain.
\end{abstract}

Observational analysis of over forty cities shows a reduction up to $60 \%$ in $\mathrm{NO}_{2}$ and up to $40 \%$ in $\mathrm{PM}_{2.5}$ but with regional differences. For example, air pollution concentrations in some cities shows an increase in $\mathrm{PM}_{2.5}$ which was attributed to mainly long range transport. A comparison with WHO Guidelines shows that for most cities, the $\mathrm{NO}_{2}$ decrease during the lockdown periods leads to meeting the annual guideline value. For $\mathrm{PM}_{2.5}$, the improvements are smaller and concentrations remain above guidelines for many regions, especially China, India, S Korea, Latin America. The observational results are corroborated by the modelling analysis conducted for the UK. Model predictions for $\mathrm{S} 1$ reveal a reduction in $\mathrm{NO}_{2}$ of about $30-40 \%$ in urban and $20-40 \%$ in rural areas. In the case of $\mathrm{PM}_{10}$ and $\mathrm{PM}_{2.5}$ model predicts about $20 \%$ reductions in urban locations and about $15 \%$ in rural areas. The reduction in $\mathrm{PM}_{10}$ is in part due to the reduction in the coarse fraction ( $\mathrm{PM}_{10}-$ $\mathrm{PM}_{2.5}$ ) of up to $14 \%$ in urban areas. There are some small increases predicted in $\mathrm{O}_{3}$ concentrations near airports and urban areas, where levels of nitrogen dioxide are significantly reduced. Analysis 
The 3rd International Electronic Conference on Atmospheric Sciences (ECAS 2020), 16-30 November 2020;

Sciforum Electronic Conference Series, Vol. 3, 2020

of S2 shows that most of the changes during the lockdown period can be attributed to reduction in road traffic emissions.

The study is currently being extended to (i) link changes in air pollutant concentrations to emissions changes, (ii) identifying regional differences across the globe and (iii) identifying lessons learnt for transitioning to lower air pollution emissions and improve air quality in global cities.

Note: no results of this study can be circulated or reported without the express opinion of Professor Ranjeet $S$ Sokhi, Chair of WMO/GAW GURME.

Keywords: air quality; COVID; pandemic; meteorology; modelling; observations; WMO; GAW

(C) 2020 by the authors. Submitted for possible open access publication under the terms and conditions of the Creative Commons Attribution (CC BY) license (http://creativecommons.org/licenses/by/4.0/). 